\title{
Hepatitis C: Genotyping
}

\author{
Norma de Paula Cavalheiro \\ Laboratory of Hepatitis, LIM 47 DMIP-HC-FMUSP; São Paulo, SP, Brazil
}

The heterogeneity of the hepatitis $\mathrm{C}$ virus (HCV) genome was described at the beginning of the 1990s. Subsequently, genotyping tests were developed in order to delineate and differentiate these variants, leading to various classifications in different parts of the world. However, in 1994, in a consensus publication, criteria were established and a universal classification was consolidated, comprising the identification of 6 large groups or viral genotypes, in addition to over 70 different subtypes distributed worldwide. It was established that genotypes differ from one another in 31\% to 33\%, as do the subtypes in $20 \%$ to $25 \%$.

The standardization in HCV classification was in accordance with the uniformity of data published in scientific and epidemiological studies on evolution and pathogenesis. Due to genomic peculiarities of different populations in the world, databases for centralization and collection of information on HCV circulating genomes were created, by region: the first in Japan by Prof. Masashi Mizokami et al. (http://s2as02.genes.nig.ac.jp/); the second in the European Union by Prof. Gilbert Deleage et al. (http://euhcvdb.fr/); and the third in the United States by Dr. Carla Kuiken et al. (http:/ /hcv.lanl.gov/ or http://hcv-db.org). The accessibility of these databases, which are consulted and updated by researchers worldwide, helps standardize terms for HCV viral genotypes and subtypes [1].

Prior to 1994, new variants were identified in Vietnam, Thailand, Burma and Indonesia. These viral genotypes were originally classified as genotypes 7, 8, 9, 10 and 11. These variants were reclassified in 2005; genotype 10a came to be denominated subtype $3 \mathrm{k}$, and genotypes $7 \mathrm{a}, 7 \mathrm{~d}, 7 \mathrm{~b}, 7 \mathrm{e} / 7 \mathrm{c}$, 11a, 9a, 9b, 9c, 8b and 8a became subtypes 6e, 6c, 6d, 6f, 6g, 6h, 6i, 6j, 6k and 6l, respectively [1].

In Brazil, HCV genotype 1 is predominant, being identified in $70 \%$ of the infected population, followed by genotypes 3 (in 25\%) and 2 (in approximately 5\%). In the southern region, the profile is differentiated, the prevalence of genotype 3 being comparable to that of genotype 1 [2,3].

The HCV viral genotype can be determined in a clinical sample in different forms, and the regions of HCV genome considered appropriate include the core, E1, NS4 and NS5 regions, as well as the 5'UTR, as mentioned in innumerable studies $[4,5]$.

The most direct method, considered the gold standard, is the sequencing of the $\mathrm{HCV}$ genome in a certain region, sufficiently divergent for different genotypes and subtypes to be distinguished [5].

The Brazilian Journal of Infectious Diseases 2007;11 (5) Suppl. 1:25-27. (C) 2007 by The Brazilian Journal of Infectious Diseases and Contexto Publishing. All rights reserved.
In practice, there are two categories of assays for the diagnosis of HCV viral types: serotyping and genotyping [6,7].

The enzyme-linked immunosorbent assay, also known as the indirect method (serotyping), commercialized only for research, discriminates among the six HCV genotypes, and not the subtypes. It presents genotype-specific antibodies for different HCV genotypes (Serotype HCV 5'NC - AbbottMurex) in the NS4 region of the genome. This test omits the determination of the quantity of circulating viral particles and presents lower sensitivity and specificity in the diagnosis of $\mathrm{HCV}$ genotypes, when compared with genotyping. Its performance is also limited in the evaluation of immunocompromised patients [3,7,8].

Genotyping (the direct method) analyzes the sequences of various regions of the genome in the differentiation of HCV genotypes and subtypes. Therefore, it depends on samples that present viral particles for analysis. These techniques are: sequencing, hybridization with genotype-specific probes, and real-time polymerase chain reaction (PCR) $[5,7,9]$.

Reverse hybridization, distributed under the commercial name Line Probe Assay (LIPA - Versant HCV Genotype assay 1.0/2.0; Innogenetics, Ghent, Belgium; distributed by Siemens Medical Solutions Diagnostics, Tarrytown, NY, USA), as described by Lieven Stuyver et al. in 1993, uses nitrocellulose tapes, on which oligonucleotide probes of region 5' NC, complementary to each specific HCV genotype/subtype, are immobilized. These tapes are hybridized under high stringency conditions, and the PCR product is marked with biotinylated primers in the amplification process. After hybridization, an avidin conjugate will bind to the biotinylated hybrid. The substrate will yield the formation of a colored product, which will be deposited on the tape and reveal the viral genotype present in the sample. Genotypes 1 through 6 , as well as subtypes 1a, 1b, 1a/1b, 2a/2c, 2b, 3a-c, 4a-h, 5a, 6a and 10a, are discriminated. The results are evaluated through visual assessment.

A new version of LIPA (version 2.0) adds probes of the core region of $\mathrm{HCV}$ and enables distinction of subtypes 1c of 6 and subtypes $1 \mathrm{a}$ and $1 \mathrm{~b}$, which, in the former version, was not possible for all the samples analyzed, since region 5' $\mathrm{NC}$ is extremely conserved and may not present sufficient diversity for the distinction of these subtypes. The interpretation of results is also performed by assessing the nitrocellulose tapes with a scanner, and the images are analyzed using a computer program. The new version is in the registration phase for distribution in Brazil [7,10,11].

Direct sequencing is the most complete information on the variations of the sequences analyzed. In addition, it is an ideal and definitive method for the study of viral genetic variability. Moreover, the study of viral dynamics in the context 
of quasispecies, during the natural history of the disease or as consequence of antiviral therapy, depends principally on the direct sequencing of certain regions of the HCV genome. Furthermore, it is considered the gold standard for determining HCV genotypes and subtypes [7].

The sequencing technique for HCV genotyping consists of PCR amplification of part of the viral genome, especially of the 5'NC, NS5B and core regions. These regions are sufficiently conserved for the development of reliable primers and, at the same time, present diversity for the discrimination of viral genotypes and subtypes. The PCR employed for sequencing comes from PCR-HCV qualitative or quantitative PCR HCV products, the volume of DNA and the high purity of the resulting tapes being essential for the success of the analyses. This second PCR is performed specifically to adjust or mark these products for reading in sequencers. The assessment of both tapes, positive and negative, is essential in order to resolve possible ambiguities during the evaluation of the sequencings [7].

The sequences provided by the equipment should be analyzed, and there are international databases available for consultation, for this purpose. These databases are also used for the centralization and collection of sequences worldwide. The database recommended for Brazil is the European site http://euhcvdb.fr/, probably because of the epidemiological pathway followed during the Brazilian colonization period.

The genotyping test TRUGENE-SIEMENS HCV 5'NC Genotyping Kit (Siemens Medical Solutions Diagnostics, Tarrytown, NY, USA) offers software used in conjunction with the equipment, with a previously selected genomic library that analyzes the sequences of region 5' NC immediately after sequencing. In addition to the genotyping results, it presents the homology with patterns of HCV genotypes and subtypes based on region 5'NC. This methodology is only available to researchers $[1,7,12]$.

The real time PCR-HCV method is a quantitative molecular diagnostic test that uses TaqMan technology. In this test, genotype-specific probes marked for HCV typing were employed in order to identify genotypes 1, 2 and 3a. Another test, also based on TaqMan technology, can identify genotypes 1 to 4 and presents specific probes for subtypes $1 \mathrm{a} / \mathrm{b}, 2 \mathrm{a} / \mathrm{b} / \mathrm{c}, 3 \mathrm{a}$ and $4 \mathrm{~b} / \mathrm{c} / \mathrm{d}$. Both methodologies are based on region 5'NC [9,13-16].

Commercially, but only for research, there is the Abbott Real-Time PCR HCV Assay (Abbott Diagnostics Europe, Wiesbaden, Germany) test, which also determines the viral genotype of the samples for HCV genotypes 1a, 1b, 2a, 2b, 3, 4, 5 and 6. This methodology for the genotyping of $\mathrm{HCV}$ is associated with viral quantification, levels below $6053 \mathrm{IU} / \mathrm{mL}$ compromising the efficiency and sensitivity of the test [9].

The identification of genotypes is clinically important, and treatment protocols recommend that genotype information be obtained in order to delineate the duration and type of the medication to be used. The literature mentions that genotypes
1 and 4 are considered more resistant than are genotypes 2 and 3, and that the standard treatment consists of interferon combined with ribavirin. Another disadvantage of genotypes 1 and 4 is that they present a worse prognosis of evolution of the disease. For treatment-naïve patients, infection with genotype 1,4 or 5 should be treated for 12 months, compared with 6 months for infection with genotype 2 or 3. The Consensus Group recommends pegylated interferon combined with ribavirin for genotype 1 . For re-treatment, pegylated interferon associated with ribavirin is recommended, regardless of the genotype, in the usual doses, respecting the duration recommended for genotype 1 for other genotypes. In cases of HIV/HCV co-infection, the response to antiviral therapy for HCV is not favorable: $14 \%-38 \%$ for genotype 1 ; and $43 \%-73 \%$ for genotypes 2 and $3[1,7,17,18]$. The advantages of genotyping methods include reliability and the opportunity to obtain important information on the molecular pathogenesis of HCV [7].

The impact of HCV heterogeneity and its different genotypes on the everyday clinical management of $\mathrm{HCV}$ chronic infection has not been completely established, nor has its role as an epidemiological marker been clarified. The sensitivity and specificity of serological and virological tests can also be influenced by the heterogeneity of HCV, which justifies constant evolution in the study of patients and differentiation techniques of HCV genotypes and subtypes [4].

\section{References}

1. Simmonds P., Bukh J., Combet C., et al. Consensus proposals for a unified system of nomenclature of hepatitis $\mathrm{C}$ virus genotypes. Hepatology 2005;42:962-73.

2. Campiotto S., Pinho J.R.R., Carrilho F.J., et al. Geographic distribution of hepatitis $\mathrm{C}$ virus genotypes in Brazil. Brazilian Journal of Medical and Biological Research 2005;38:41-9.

3. Cavalheiro N.P., Barone A.A., Tengan F.M. HCV Serotypes in brazilian patients. Int J Infect Dis 2002;6:228-32.

4. Pawlotsky J.-M. Diagnostic tests for hepatitis C. J Hepatol 1999;31 Suppl. 1:71-9,.

5. Laperche S., Lunel F., Izopet J., et al. Comparison of Hepatitis C Virus NS5b and 5_ Noncoding Gene Sequencing Methods in a Multicenter Study. Journal of Clinical Microbiology 2005:733-9.

6. Pawlotsky J.-M., Prescott L., Simmonds P., et al. Serological determination of hepatitis $\mathrm{C}$ virus genotype: Comparison with a standardized genotyping assay. J Clin Microbiol 1997;35:1734-9.

7. Stéphane C., Pawlotsky J.-M. Hepatitis C virus: Virology, diagnosis and management of antiviral therapy. World J Gastroenterol 2007;13(17):2461-6.

8. Prescott L.E., Simmonds P. Serological genotyping using synthetic peptides derived from the NS4 region. In: LAU, JOHNSON YIU-NAM. Hepatitis C protocols: methods in molecular medicine. Totowa, Humana Press, cap.17, p.199-205, 1998.

9. Raymond H.W., Carol Cimmins. Evaluation of the Abbott Molecular Diagnostics Real Time PCR Assay for HCV Quantitative Viral Load and HCV Genotyping. Poster S30, Clinical Virology Symposium, 2004.

10. Nadarajah R., Khan Y., Miller S.A., Brooks G.F. Evolution of a new generation Line-Probe Assay that detects 5'untranslated and Core regions to genotype and subtype Hepatitis C Virus. Am J Clin Pathol 2007;128:300-4. 
11. Bouchardeau F., Cantaloube J.F., Chevaliez S., et al. Improvement of Hepatitis C Virus (HCV) Genotype Determination with the New Version of the INNO-LiPA HCV Assay. Journal of Clinical Microbiology 2007;45:1140-5.

12. Nolte F.S., Green A.M., Fiebelkorn K.R., et al. Clinical evaluation of two methods for genotyping Hepatitis $\mathrm{C}$ virus based on analysis of the 5'noncoding region. Journal of Clinical Microbiology 2003;41:1558-64.

13. Lindh M., Hannoun C. Genotyping of hepatitis C virus by Taqman real-time PCR. Journal of Clinical Virology 2005;34:108-14.

14. Moghaddam A., Reinton N., Dalgard O. A rapid real-time PCR assay for determination virus genotypes 1, 2 and 3a. Journal of Viral Hepatitis 2006;13:222-9.
15. Rolfe K.J., Alexander G.J.M., Tim G.W., et al. A real-time Taqman method for hepatitis $\mathrm{C}$ virus genotyping. Journal of Clinical Virology 2005;34:115-21.

16. Mukaide M., Tanaka Y., Kakuda H., et al. New combination test for hepatitis $\mathrm{C}$ virus genotype and viral load determination using Amplicor GT HCV MONITOR test v2.0. World J Gastroenterol 2005;11(4):469-75.

17. Poynard T., Marcellin P., Lee S.S., et al. Randomized trial of interferon alfa 2 b plus ribavirin for 48 weeks or for 24 weeks versus interferon alfa 2bplus placebo for 48 weeks for treatment of chronic infectious hepatitis C virus. Lancet 1998;352:1426-32.

18. Tom Wong, Samuel S.L. Hepatitis C: a review for primary care physicians. CMAJ 2006;174(5):649-59. 